\title{
REFLEXÕES SOBRE REPRESENTAÇÕES ESPACIAIS DE ÁRVORES DE RUA EM AMBIENTE DE SISTEMA DE INFORMAÇÕES GEOGRÁFICAS
}

\author{
REFLECTIONS ON SPACE REPRESENTATIONS OF STREET TREES IN A \\ GEOGRAPHICAL INFORMATION SYSTEM ENVIRONMENT
}

\author{
Pedro Paulo Gomes de Oliveira' ${ }^{1}$ Paulo Costa de Oliveira Filho²
}

\section{RESUMO}

Estudos sobre arborização viária no Brasil ainda são recentes, por tratar-se de uma prática relativamente nova, tendo em vista a complexidade que envolve o planejamento da arborização urbana, se faz necessário a utilização de ferramentas que possam subsidiar essa atividade. Desta forma o Sistema de Informações Geográficas se tornou uma ferramenta base para o planejamento da arborização urbana, por meio dele é possível obter suporte espacial e fazer consultas referentes ao manejo e monitoramento das árvores de rua. Atualmente utiliza-se a representação da unidade espacial "árvore" em ambiente SIG, como uma representação espacial pontual ou até mesmo poligonal em função da escala de trabalho. Esse modelo atende à representação dos objetos para associação a bancos de dados, porém, apresentam dificuldades do ponto de vista de implementação em função da quantidade de objetos a serem associados ao manejo das árvores, o qual é voltado normalmente para grupos de árvores, sujeitas às mesmas condições de manejo. Assim sendo, a individualização das árvores de rua pode não ser a melhor forma de organizar um modelo espacial em SIG. Este estudo propõe apresentar reflexões sobre um sistema de representação linear de árvores de rua como alternativa para espacializar unidades de manejo em ambiente SIG.

Palavras-chave: SIG; Arborização urbana; Unidade de manejo linear.

\section{ABSTRACT}

Studies on road tree planting in Brazil are still recent, for it is a relatively new practice, considering the complexity involved in urban tree-planting planning, it is necessary to use tools that can subsidize this activity. In this way, the Geographic Information System has become a basic tool for the urban afforestation planning, through which it is possible to obtain space support and make queries about the management and monitoring of the trees in the street. Currently, the representation of the space unit "tree" in GIS environment is used, as a punctual or polygonal space representation according to the work scale. This model responds to the representation of the objects for association to databases, however, it shows difficulties implementation due to amount of objects to be associated to the management of the trees, which is normally focused to groups of trees, issued to same management conditions. Thus, individualization of street trees may not be a better way to organize a spatial model in GIS. This study proposes to present reflections on a system of linear representation of street trees as an alternative to spatialize management units in a GIS environment.

Keywords: GIS, Urban tree-planting, Linear management unit.

Recebido em 05.03.2018 e aceito em 18.05.2018

1 Engenheiro Florestal, Mestrando do Programa de Pós-Graduação em Ciências Florestais da Universidade Estadual do CentroOeste. Irati/PR. Email: pedropaulo_@hotmail.com

2 Engenheiro Florestal, Profํㅡㄹ Dro da Universidade Estadual do Centro-Oeste. Irati/PR. Email: paulocostafh@irati.unicento.br 


\section{INTRODUÇÃO}

As árvores de rua, como componentes das áreas verdes urbanas, apresentam como condição extraordinária o fato de que sua existência independe de questões de direito de propriedade normalmente incidentes na maioria das áreas verdes existentes nas cidades brasileiras.

Esta característica a difere das poucas áreas verdes ainda presentes em tecido urbano, sobretudo em cidades não planejadas, onde as gestões municipais demonstram dificuldades para contemplar questões como expansão de áreas construídas e seus impactos decorrentes da substituição de áreas verdes privadas.

Considerando que grande parte da população vive nas áreas urbanas no Brasil, não é difícil imaginar o número de obstáculos que a arborização de ruas precisa superar para seu bom desenvolvimento, isto baseado no fato de que as árvores, no meio urbano, estão em condições adversas do local de sua procedência que é a floresta.

A obtenção de resultados satisfatórios exige que todas as atividades desenvolvidas em um município sejam planejadas. Neste contexto, a arborização urbana merece atenção especial, pois ruas arborizadas sem nenhum critério trazem prejuízos ao poder público, além de inúmeros transtornos causados pela falta de planejamento e conhecimento dos que norteiam a arborização de ruas (SCHUCH, 2006).

De acordo com este raciocínio, a arborização bem planejada é muito importante independente do porte da cidade, pois, é muito mais fácil implantar quando se tem um planejamento, do que tentar se encaixar dentro das condições já existentes e solucionar problemas de toda ordem, caso contrário, passa a ter um caráter de remediação (SILVA; MORAES, 2016).

Segundo Raber e Rebelato (2010) para um adequado planejamento da arborização das ruas e avenidas de uma cidade, alguns fatores devem ser considerados, sendo eles: seleção de espécies, implantação, manutenção e monitoramento.

Os estudos sobre arborização viária no Brasil ainda são recentes e desordenados, por tratar-se de uma prática relativamente nova, e pelo fato de ser considerado um problema de menor importância no planejamento urbano (SCHUCH, 2006).

Tendo em vista a complexidade que envolve o planejamento da arborização urbana, se faz necessário a utilização de ferramentas que possam subsidiar essa atividade.

Dessa forma, o Sistema de Informação Geográfica - SIG tem-se mostrado como importante ferramenta, possibilitando criar de forma prática e rápida, bancos de dados e documentos cartográficos com as mais diversas informações que servirão de base para o adequado planejamento. As informações contidas no mundo real são mapeadas nos SIGs dentro 
de estruturas matricial e/ou vetorial, as imagens são representadas no formato matricial e objetos geográficos são representados no formato vetorial

As duas formas básicas de representação dos dados espaciais em um SIG são as formas vetorial e matricial. A representação em formato matricial é caracterizada por uma matriz de células também chamadas de pixels, ou seja, o dado matricial é a imagem utilizada para representar as feições da superfície geográfica, as imagens obtidas de sensores orbitais, aerotransportados e modelos digitais de terreno são naturalmente representadas no formato matricial.

A representação em formato vetorial utiliza pontos, linhas e polígonos para representar a geometria das entidades geográficas, entidades geográficas lineares, como ruas, divisões político-administrativas, redes de tráfego e hidrografia, são naturalmente representadas em formato vetorial.

O processo de implantação do SIG visa facilitar a coleta e análise dos dados obtidos, a integração de dados de diversos formatos, o fornecimento de informações e a atualização e recuperação facilitada dos dados (OLIVEIRA FILHO; SILVA, 2010; LIMA NETO; BIONDI, 2012).

As informações gráficas originárias do cadastro e dos censos (dados geográficos), como as informações (dados alfanuméricos) que fazem parte de informações relacionadas à gestão municipal urbana, associado a um sistema geográfico de informação fornecem informações essenciais para o planejamento e a gestão territorial (DIAS et al., 2016).

Este estudo se justifica primeiro por contribuir com a gestão pública por apresentar e ilustrar um método que permite apoiar as atividades de planejamento urbano articulado ao planejamento de arvores urbanas, apresentando reflexões sobre um sistema de representação linear de árvores de rua como alternativa para espacializar unidades de manejo em um sistema de informações geográficas.

\section{MATERIAL E MÉTODOS}

\section{Localização da área de estudo}

O município de Irati, está localizado na região centro sul do estado do Paraná, no paralelo $25^{\circ} 27^{\prime} 56^{\prime \prime}$ de latitude sul com interseção com o meridiano $50^{\circ} 37^{\prime} 51^{\prime \prime}$ de longitude oeste, a uma altitude de $812 \mathrm{~m}$, em relação ao nível do mar, a cerca de $150 \mathrm{~km}$ de Curitiba, capital do estado (Figura 1). A área do município é de $999,519 \mathrm{Km}^{2}$, com uma população de 56.207 habitantes, sendo 44.932 habitantes da área urbana (79,94\%) e 11.275 habitantes da área rural. 


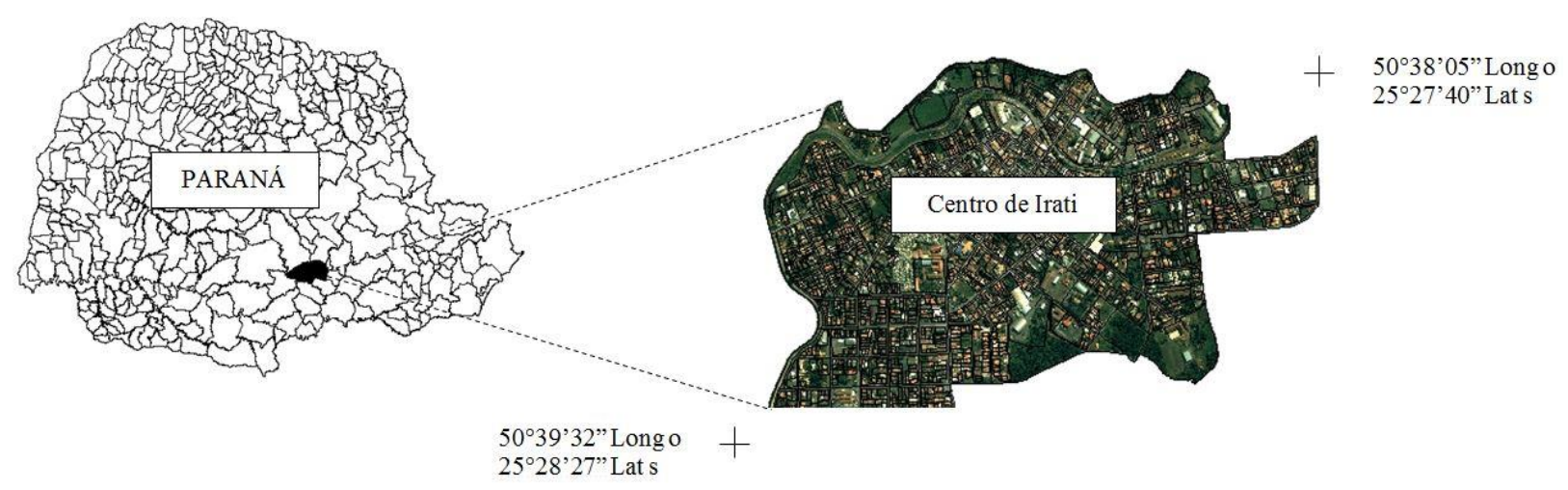

Figura 1. Localização geográfica da área de estudo

Figure 1. Geographical location of the study area

Para o desenvolvimento do presente estudo foram realizadas simulações de cenários alternativos para representar as unidades amostrais de arvores de rua, utilizando o software QGIS 2.14.10, com o objetivo de verificar o efeito das operações de manejo florestal urbano utilizando o método de representação espacial linear.

\section{Cenários Avaliados}

As simulações foram feitas com base em quatro cenários, conforme a Tabela 1. Para a definição dos cenários foram consideradas as principais práticas de manejo realizadas em arvores urbanas.

As simulações foram realizadas utilizando como base o município de Irati - PR. As informações foram fornecidas pela Secretaria de Ecologia e Meio Ambientes através de arquivos vetoriais no formato DWG, contendo a base cartográfica com os limites dos bairros do município, eixo das ruas, meio fio, nomenclatura das ruas, lotes, instalações subterrâneas, localização dos postes e das árvores e dados do levantamento da arborização das vias públicas.

A estruturação do modelo de dados orientado ao objeto linear de representação em ambiente SIG foi à primeira etapa do trabalho, sendo realizada por meio da importação e organização dos arquivos vetoriais e associação a atributos normalmente utilizados no manejo da arborização urbana.

Para associar as árvores espacializadas com os seus respectivos dados de levantamento de campo utilizou-se o modelo linear de representação da unidade de manejo, sendo considerado um grupo de árvores de mesma idade e espécie. 
Tabela 1. Cenários propostos de manejos florestais urbanos para a avaliação da representação espacial linear

Table 1. Proposed urban forest management scenarios for the evaluation of linear spatial representation

Cenários
Cenário 1: Classificação das espécies
florestais

Descrição

Composições florísticas de espécies florestais ao longo de avenidas e ruas em área urbana, classificadas em grupos.

Cenário 2: Classificação de idade

Agrupamento de arvores urbano, com as mesmas idades, independente do grupo de espécie florestal.

Cenário 3: Manejo - Poda de formação

Usada na fase jovem da árvore, através do corte de galhos mais finos, visando a obtenção de uma copa bem conformada, respeitando o modelo arquitetônico da espécie e adequado às características do local de plantio. Seu objetivo é orientar o crescimento da copa da árvore, eliminando precocemente os galhos.

Cenário 4: Manejo - Poda de manutenção / Substituição / Operações Realizadas
Usada na fase adulta da árvore, buscando evitar eventuais quebras de galhos secos ou mal formados ou para manter a convivência da copa com os equipamentos urbanos instalados no seu entorno.

A substituição de arvores é aplicada quando existe a necessidade de trocar o indivíduo.

Desta forma considerou-se a representação vetorial, linear flexível às mudanças de direção ou cruzamentos, considerando ruas e quadras, permitindo então que cada segmento linear possa representar todo um grupo de árvores ou unidade de manejo independentemente.

\section{RESULTADOS E DISCUSSÃO}

Conforme citado na metodologia, após a implementação do banco de dados orientado ao objeto linear de representação, algumas simulações foram realizadas para testar a eficiência do modelo.

A figura 2 apresenta as simulações sobre os temas que fazem parte das intervenções de manejo da arborização urbana: classificação das espécies, classificação de idade, poda de formação, podas de manutenção, substituição de indivíduos e operações realizadas. 

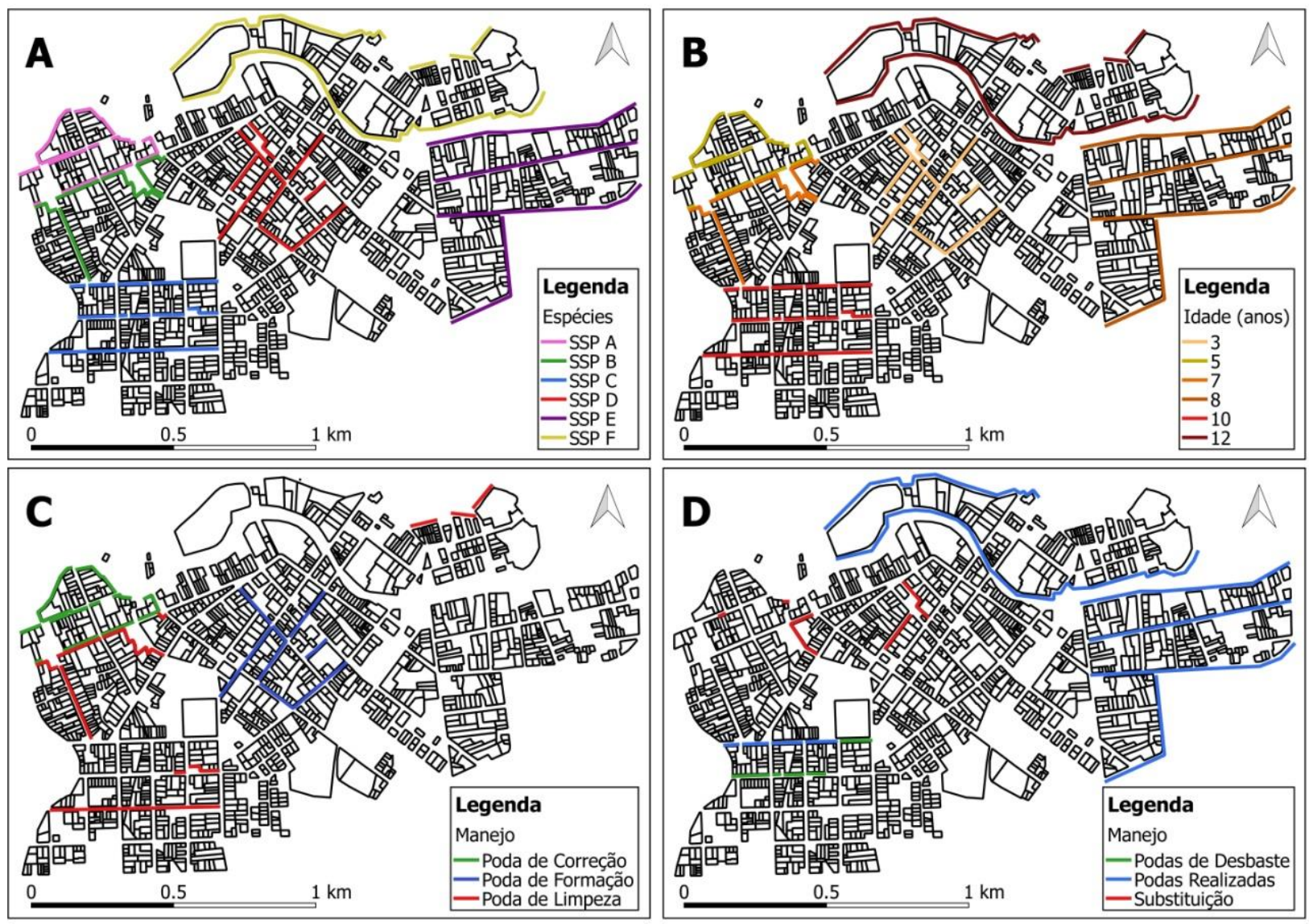

NOTA: Simulação da classificação de espécies florestais (Figura 2a) Simulação da classificação de idade (Figura 2b) Simulação da programação de podas de formação (Figura 2c) Simulação da programação de podas de manutenção (Figura 2d)

Figura 2. Simulação de atividades operacionais de manejo da arborização urbana

Figure 2. Simulation of operational activities of urban afforestation management

Conforme apresentado na figura 2, cada unidade de manejo pode ser representada com uma linha, sem qualquer limitação à flexibilidade, para que a mesma represente de fato o que é a unidade de manejo, ou seja, agrupamento linear de árvores de mesma espécie e mesma idade, às quais se pretende condicionar às mesmas intervenções silviculturais.

Segundo Archela e Théry (2008), os mapas de lineares são indicados para representar feições que se desenvolvem linearmente no espaço, por isso, pode ser reduzida a forma de uma linha.

Segundo Campo Grande (2010) e o manual da Cemig (2011), a escolha de uma só espécie para cada rua ou cada lado de rua, facilita o acompanhamento de seu desenvolvimento, o controle de pragas e doenças e o programa de podas.

Visando o planejamento de novas áreas de florestas urbanas, este método de plantio seria o mais adequado para uma representação das unidades amostrais de forma linear, havendo ao longo de uma via, uma única espécie, com as mesmas idades, submetidas aos mesmos tratamentos silviculturais. Mas não existe uma metodologia considerada de uso universal para essas representações. 
Geralmente tem sido utilizada a representação pontual, já citada por diversos autores: Spadotto e Delmanto Júnior (2009) utilizaram um modelo de representação espacial pontual onde cada árvore foi associada aos seus atributos para a gestão da arborização no perímetro urbano do bairro Jardim Paraíso em Botucatu-SP, a partir da interpretação de uma fotografia aérea de escala 1:30.000.

Oliveira Filho e Silva (2010) implementaram um modelo de dados protótipo direcionado para a gestão da arborização urbana da região central de Guarapuava, Paraná. O modelo foi orientado com representação espacial pontual, onde cada ponto representava uma árvore, e a partir da associação de atributos e à espacialização de componentes da infra-estrutura urbana, tais como logradouros, rede de água e esgoto, rede elétrica entre outros, foram realizadas várias consultas espaciais e lógicas que permitiram simular maior agilidade ao manejo da arborização urbana.

Tanto Spadotto e Delmanto Júnior (2009) quanto Oliveira-Filho e Silva (2010) utilizaram um objeto de representação pontual, ou seja, com o único propósito de espacializar o posicionamento de cada árvore sem apresentar detalhes da forma da copa. Esse tipo de representação pode ser espacializada a partir de um ponto central para ser expandido através de uma operação simples de faixas de distância para manter um padrão do elemento de representação, sem perder de foco o centróide definido pelo posicionamento mais próximo do tronco.

Lima Neto e Biondi (2012) trabalharam com fotografias aéreas em escala de 1:8.000 para delinear e vetorizar os limites das copas das árvores de rua em três bairros de Curitiba. Neste trabalho o objetivo foi à obtenção de algumas métricas tais como área de copa e comparação com medições convencionais realizadas em campo. Os mesmos autores utilizaram um modelo de representação poligonal irregular possível de ser obtido devido à escala das fotos e também à qualidade das mesmas na capacidade de ampliação em ambiente digital.

Neste trabalho, em que os autores utilizaram um modelo de representação poligonal irregular vetorizado por interpretação dos limites das copas nas imagens, a representação temática visualizada fornece a informação da cobertura proporcionada pela arborização de rua no espaço urbano.

Mayer, Oliveira Filho e Bobrowski (2015) utilizou um SIG para espacializar algumas áreas amostrais contendo quadras com ruas e o posicionamento de árvores de rua, representadas através de um objeto pontual e homogêneo para analisar as relações ou conflitos entre as árvores e as estruturas urbanas através de ferramentas de análise espacial, com o propósito de auxiliar o planejamento e gestão da arborização de vias públicas.

Esse modelo apresentado por Mayer et al., (2015) contendo diferentes composições de espécies apresenta-se como ideal para uso de representação linear de unidades de manejo em 
SIG, pois ruas ou segmentos de ruas são projetadas com grupos de árvores com a mesma espécie e idade, portanto mesmas condições de manejo.

Modelos como esses apresentados, têm trazido alguma evolução quando realizam associação de dados alfanuméricos de importância ao manejo da arborização urbana aos elementos gráficos de representação das árvores.

A representação espacial pontual, onde, cada ponto representa uma árvore, pode ser realizada a partir de um ponto central definido pelo posicionamento mais próximo do tronco ou através da interpretação de fotografias aéreas ou imagens orbitais de altíssima resolução.

A principal vantagem deste tipo de representação é a associação de cada indivíduo as suas variáveis dendrométricas. Apesar da representação pontual abordar os atributos de modo individual, talvez não haja necessidade de que a informação seja relacionada por árvore, mas sim por grupo de árvores sob as mesmas condições de manejo.

A representação das informações contidas no mundo real, no entanto, é complexa demais para permitir uma representação completa. Desta forma, é necessário construir uma abstração dos objetos e fenômenos do mundo real, de modo a obter uma forma de representação adequada, embora simplificada, que seja correspondente às finalidades das aplicações do banco de dados.

A individualização da árvore como geo-objeto de representação espacial induz a implementação de bases de dados contendo grande volume de entidades a serem associadas a tabelas de atributos. Isso dificulta bastante a manipulação de dados sobre o manejo.

A individualização da árvore como estratégia para dar subsídio espacial ao manejo das árvores de rua pode não ser a melhor forma de organizar um modelo espacial em sistema de informações geográficas.

Uma das justificativas para fundamentar o uso da representação linear, está no fato de que técnicas de gestão da arborização urbana procuram manter unidades de manejo contendo indivíduos de mesma espécie submetidas às mesmas condições de manejo, estas, normalmente são combinadas a outras por meio de composições florísticas.

Relacionando os dois métodos de representação das florestas urbanas, submetidos as mesmas condições, podemos observar que a representação pontual apresenta uma maior quantidade de informação pois cada ponto representa uma árvore que será atribuído informações com relação a cada ponto $A$ representação linear esse número de informação é reduzido pois teríamos uma linha que representa a unidade amostral

Desta forma reduziríamos o número de informação contido no bando de dados, o que facilitaria as analises, consulta e visualização

Dessa forma as unidades de manejo lineares são mais flexíveis, capaz de representar agrupamentos de árvores que estão na mesma rua ou em mais ruas podendo circundar a quadra, 
podendo transpassar um cruzamento e continuar na mesma rua e em outra quadra, podendo haver ou não a quebra da linha da unidade de produção em função do manejo (Figura 3).

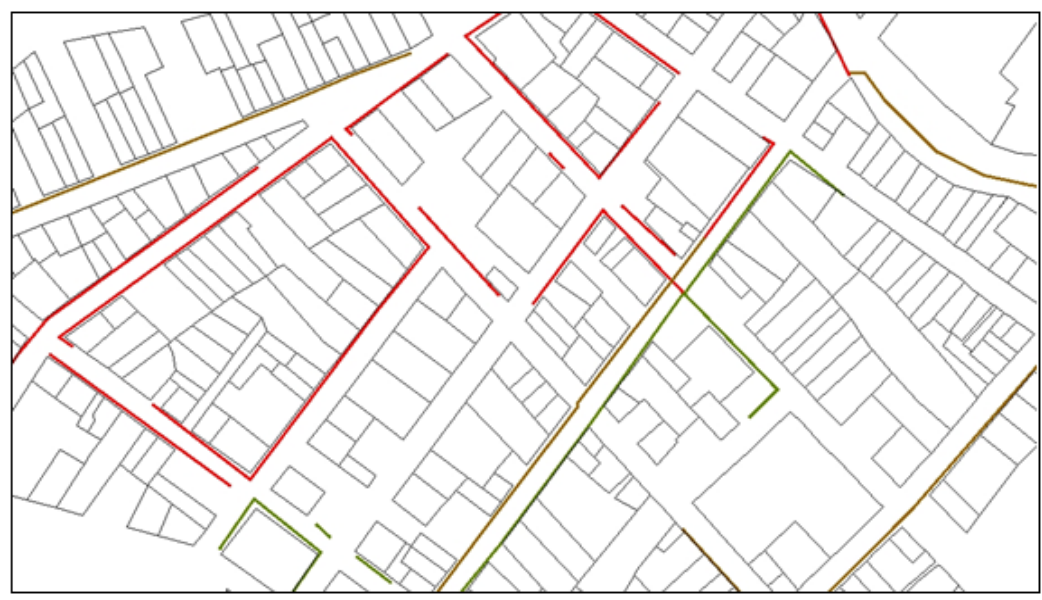

Figura 3. Representa espacial linear das unidades de manejo

Figure 3. Linear spatial representation of the management units

\section{CONCLUSÕES}

A utilização de representações lineares em ambiente SIG pode reduzir significativamente o número de geo-objetos a serem associados aos atributos, facilitando implementação e a manipulação dos dados.

A representação da unidade "árvore" torna-se bastante complicada do ponto de vista de atualização no banco de dados devido à quantidade de árvores existentes em qualquer modelo urbano espacializado. Este tipo de aplicação de um modelo de dados orientado a um objeto em um SIG pode ser testado com mais agilidade se reduzido imensamente o número de unidades de manejo ou gerenciamento e associação aos atributos.

A representação linear para unidades de manejo de árvores de rua pode trazer mais facilidades na implementação de sistemas de informações geográficas mais direcionadas a esta atividade, favorecendo o planejamento e a tomada de decisão com base no controle espacial da atividade. 


\section{REFERÊNCIAS}

ARCHELA, R. S.; THÉRY, H. Orientação metodológica para construção e leitura de mapas temáticos. Revista Franco-Brasileira de Geografia, [online] v.3, 2008. 21p.

\section{CAMPO GRANDE - PREFEITURA MUNICIPAL. Plano Diretor da Arborização Urbana de Campo Grande. Campo Grande, 2010. 158p}

COMPANHIA ENERGÉTICA DE MINAS GERAIS (CEMIG). Manual de Arborização. Belo Horizonte: CEMIG, 2011. 112p.

DIAS, P.; PELEGRINA, M. A.; JULIÃO, R. P.; BERTOTTI, L. G. Análise exploratória de estatística espacial aplicada ao espaço urbano. Ambiência, Guarapuava, v.12, n.2, p. 539-549, 2016.

LIMA NETO, E. M; BIONDI, D. Detecção de árvores de ruas da cidade de Curitiba, PR, utilizando fotografias aéreas. Revista Brasileira de Ciências Agrárias, Recife, v. 7, n. 4, p. 641-647, 2012.

MAYER, C. L. D.; OLIVEIRA FILHO, P. C. de; BOBROWSKI, R. Análise espacial de conflitos da arborização de vias Públicas: caso Irati, Paraná. Revista Floresta, Curitiba, v. 45, n. 1, p. 11-20, 2015.

OLIVEIRA FILHO, P.; SILVA, S. V. K. Um sistema de informações para suporte espacial e de decisões à gestão da arborização urbana no município de Guarapuava, Paraná. REVSBAU, Piracicaba, v. 5, n. 3, p. 82-96, 2010.

RABER, A. P.; REBELATO, G. S. Arborização viária do município de Colorado, RS - Brasil: análise quali-quantitativa. REVSBAU, Piracicaba, v. 5, n. 1, p. 183-199, 2010.

SILVA, S. L. da; MORAES, M. V. A. R. Percepção ambiental e arborização urbana em Teresina, Piauí, Revista Equador, Teresina, v. 5, n. 3, p. 320-339, 2016.

SCHUCH, M. I. S. Arborização Urbana: uma contribuição à qualidade de vida com o uso de geotecnologias. Santa Maria, 2006. 102f. Dissertação (Mestrado em Geomática) Universidade Federal de Santa Maria, Santa Maria, 2006.

SPADOTTO, L. G. F.; DELMANTO JÚNIOR, O. Planejamento e gerenciamento da arborização urbana utilizando técnicas de geoprocessamento. Revista Tékhne $\varepsilon$ Lógos, Botucatu, v. 1, n. 1, p. 35-53, 2009. 